\title{
Incidencia de la radiación microondas en la cristalinidad de materiales laminares
}

\author{
P. BENITO, F.M. LABAJOS, V. RIVES \\ Dpto. Química Inorgánica. Universidad de Salamanca.Salamanca.
}

\begin{abstract}
Se presentan los resultados obtenidos utilizando radiación microondas en la preparación de compuestos tipo hidrotalcita de composición $\mathrm{Mg}, \mathrm{Al}-\mathrm{CO}_{3^{\prime}} \mathrm{Mg}, \mathrm{Cr}-\mathrm{CO}_{3^{\prime}} \mathrm{Ni}, \mathrm{Al}-\mathrm{CO}_{3}$ y $\mathrm{Zn}, \mathrm{Ni}, \mathrm{Al}-\mathrm{CO}_{3}$. Los materiales obtenidos se comparan con homólogos preparados por métodos convencionales. Se ha elaborado un protocolo de trabajo que permita obtener resultados reproducibles, siendo éste un aspecto novedoso de la utilización de microondas en la síntesis de sólidos inorgánicos. Los sólidos obtenidos fueron caracterizados mediante difracción de rayos $\mathrm{X}$, análisis térmico, análisis químico elemental y adsorción-desorción de $\mathrm{N}_{2}$ a $-196^{\circ} \mathrm{C}$. En todos los casos se obtuvieron compuestos tipo hidrotalcita con la composición química deseada. La cristalinidad final del sólido se ve afectada por el tiempo de exposición a la radiación microondas. Los materiales obtenidos utilizando microondas muestran una mayor homogeneidad en el tamaño de grano y un buen grado cristalino y mayor orden de las moléculas de agua interlaminares.
\end{abstract}

Palabras clave: Compuestos tipo hidrotalcita, hidrotermal, microondas.

\section{Effect of microware radiation on the crystallinity of layered materials}

The results obtained in the preparation of hydrotalcite-like compounds using microwave radiation are reported. The following systems were synthesized: $\mathrm{Mg}, \mathrm{Al}-\mathrm{CO}_{3^{\prime}} \mathrm{Mg}, \mathrm{Cr}-\mathrm{CO}_{3}, \mathrm{Ni}, \mathrm{Al}-\mathrm{CO}_{3}$ y $\mathrm{Zn}, \mathrm{Ni}, \mathrm{Al}-\mathrm{CO}_{3}$. The results obtained by the microwave-hydrotermal method have been compared with the conventional-hydrotermal one. A working protocol, which allows us to obtain reproducible results, has been elaborated, and this is a new aspect in the synthesis of inorganic solids. The obtained solids were characterized by several techniques $(\mathrm{X}$ ray difracttion, thermal analysis, chemical analysis and $\mathrm{N}_{2}$ adsorption-desorption at $\left.-196^{\circ} \mathrm{C}\right)$. Hydrotalcite-like compounds with the desirable chemical composition were obtained. The cristallinity depends on the microwave radiation exposure time in all cases. Materials prepared using microwave radiation exhibit a better grain size homogeneity and a good crystalline degree and it also influences over the water molecules arrangement in the solid.

Key words: Hydrotalcite-like compounds, hydrothermal, microwaves.

\section{1.-INTRODUCCIÓN}

Los hidróxidos dobles laminares (LDHs) son una familia de materiales laminares que han sido objeto de numerosos estudios en los últimos años (1). Su estructura se puede describir como un compuesto formado por láminas de brucita (tipo $\mathrm{CdI}_{2}$ ) en las que se han producido sustituciones isomórficas del catión divalente por el catión trivalente; el exceso de carga positiva generado se compensa con aniones que se sitúan, junto con moléculas de agua, en el espacio interlaminar. Aunque los compuestos más abundantes son aquéllos en los que están presentes metales en estado dipositivo y tripositivo, también se han preparado materiales con cationes tetravalentes y monovalentes $(2,3)$. La síntesis de estos materiales se lleva a cabo por coprecipitación de las sales de los cationes metálicos que van a formar parte de las láminas. La facilidad de este proceso ha propiciado que se hayan preparado gran cantidad de compuestos en los que se varía tanto la composición de las láminas como la de la región interlaminar (4-6). La cristalinidad de los sólidos puede mejorarse mediante el empleo de tratamiento hidrotermal durante varios días (7). La aplicación de nuevas tecnologías a la síntesis de materiales es un tema de gran actualidad por las perspectivas de mejora que suponen. Una de las técnicas que está proporcionando buenos resultados es la aplicación de radiación microondas al procesado de materiales. Debido a la naturaleza de las microondas se produce un calentamiento volumétrico, que permite mejorar las propiedades físicas, preparar nuevos materiales que no se pueden obtener por otros métodos y disminuir el impacto ambiental del proceso. Por estas razones se ha empleado para obtener diversos materiales inorgánicos (8). En este trabajo se describe la preparación de compuestos tipo hidrotalcita empleando radiación microondas y el estudio comparativo de sus propiedades con compuestos similares preparados usando métodos tradicionales.

\section{EXPERIMENTAL}

Se han preparado sólidos de composición X,Y,Z-A, donde X,Y,Z indica los metales laminares y A el anión interlaminar. La relación molar $\mathrm{M}^{2+} / \mathrm{M}^{3+}$ empleada fue 2/1 para los sistemas $\mathrm{Mg}, \mathrm{Al}-\mathrm{CO}_{3^{\prime}}, \mathrm{Mg}, \mathrm{Cr}-\mathrm{CO}_{3^{\prime}}$ $\mathrm{Zn}, \mathrm{Ni}, \mathrm{Al}-\mathrm{CO}_{3}$ y $3 / 1$ para $\mathrm{Ni}, \mathrm{Al}-\mathrm{CO}_{3}$. Los compuestos se prepararon por coprecipitación a $\mathrm{pH}$ constante (9), a partir de nitratos de los cationes metálicos y una disolución básica de $\mathrm{Na}_{2} \mathrm{CO}_{3}$ y $\mathrm{NaOH}$. El pH se fijó empleando exceso de sosa, excepto para el sistema $\mathrm{Zn}, \mathrm{Ni}, \mathrm{Al}-\mathrm{CO}_{3^{\prime}}$ para el que el $\mathrm{pH}$ se mantuvo a un valor constante de 7.5 usando un Dosimat 725 (Metrohm) acoplado a un pHmetro 691 (Metrohm). Fracciones de la suspensión inicial obtenida se sometieron a distintos procesos de envejecimiento. Una de ellas no se sometió a ningún proceso. Otra fue envejecida durante tres días en un reactor Phaxe 2000, en estufa a $125^{\circ} \mathrm{C}$. El resto de alícuotas han sido tratadas en bombas de digestión en un horno microondas, Milestone Ethos Plus, a $125^{\circ} \mathrm{C}$ durante periodos de 10, 30, 60, 180 y 300 minutos. Los precipitados fueron separados por centrifugación, lavados con agua bidestilada hasta ausencia de nitratos 
y secados en una estufa a $40^{\circ} \mathrm{C}$. Las muestras obtenidas por el método convencional se designan como XY0 las no sometidas a envejecimiento y XY3 las sometidas a tratamiento hidrotermal convencional durante tres días. Por su parte, las muestras tratadas en microondas se denominan como XYHWt, siendo t el tiempo de irradiación en minutos. Por su parte, la caracterización de los sólidos se realizó empleando las técnicas y equipos que se indican. El estudio de Análisis químico elemental se realizó mediante absorción atómica en un aparato Mark 2 ELL-240 en el Servicio General de Análisis Químico Aplicado de la Universidad de Salamanca. Los difractogramas de rayos $\mathrm{X}$ en polvo fueron registrados en un difractómetro Siemens D-500. Los análisis térmicos, termogravimétrico (TG) y diferencial (DTA), se llevaron a cabo en aparatos Perkin Elmer, TG-7 y DTA-7, a una velocidad de calentamiento de $10^{\circ} \mathrm{C} \mathrm{min}^{-1}$. Las isotermas de adsorción/ desorción de nitrógeno a $-196^{\circ} \mathrm{C}$ se realizaron en un aparato Gemini de Micromeritics, desgasificando la muestra a $150{ }^{\circ} \mathrm{C}$ durante $2 \mathrm{~h}$ en un aparato FlowPrep 060. Los espectros FT-IR fueron registrados en un espectrómetro Perkin-Elmer FT1730, soportando la muestra en $\mathrm{KBr}$.

\section{RESULTADOS Y DISCUSIÓN}

Los datos de análisis químico y difracción de rayos $\mathrm{X}$ indican que en todos los casos se obtuvieron materiales tipo hidrotalcita con relaciones atómicas muy próximas a las de las disoluciones de partida, con ligeras variaciones en la composición para los compuestos obtenidos usando microondas, debido sin duda a la distinta naturaleza de los componentes y a su interacción con la radiación microondas. Para el sistema $\mathrm{Mg}, \mathrm{Al}-\mathrm{CO}_{3}$ las diferencias se atribuyen a la descomposición de parte del material bajo la influencia del campo, redisolución del $\mathrm{Mg}^{2+}$ y precipitación del $\mathrm{Al}^{3+}$, debido a su mayor acidez, en forma de hidróxido amorfo. Para el sistema $\mathrm{Mg}, \mathrm{Cr}-\mathrm{CO}_{3}$ la relación es menor que la teórica con independencia del proceso de envejecimiento, debido a la distinta solubilidad entre $\mathrm{Mg}(\mathrm{OH})_{2}$ y $\mathrm{Cr}(\mathrm{OH})_{3}(10)$. En los compuestos $\mathrm{Ni}, \mathrm{Al}-\mathrm{CO}_{3}$ la relación es superior a tres, probablemente debido a que durante la coprecipitación se trabajó a valores de $\mathrm{pH}$ altos. Cuando se introduce zinc junto con el níquel, sistema $\mathrm{Zn}, \mathrm{Ni}, \mathrm{Al}-\mathrm{CO}_{3^{\prime}}$ los compuestos presentan mayor estabilidad frente a la radiación microondas. Por lo que respecta a la cristalinidad de los sólidos, en la Figura 1 se recoge, a modo de ejemplo, los difractogramas de los materiales del sistema $\mathrm{Mg}, \mathrm{Cr}-\mathrm{CO}_{3^{\prime}}$ donde se puede apreciar la incidencia del tratamiento hidrotermal sobre la cristalinidad de los sólidos finales. Llama la atención el notable efecto de la radiación microondas en la cristalinidad del sólido final, detectable incluso cuando los periodos de aplicación son cortos. Más espectaculares son los resultados obtenidos cuando se emplean microondas durante 300 minutos; el difractograma presenta picos de intensidades comparables a los logrados con tratamiento hidrotermal convencional durante tres días, lo que indica que se ha alcanzado prácticamente la misma cristalinidad para ambas muestras. En la Tabla I se recogen los parámetros cristalinos, calculados a partir de las posiciones de los picos (003) y (110), según las expresiones $\mathrm{c}=$ $3 c^{\prime}$, siendo $c^{\prime}=(1 / 2)\left[d_{(003)}+2 d_{(006)}\right], y a=2 d_{(110)^{\prime}}$ así como los valores de tamaño de partícula calculados a partir de la anchura de pico a altura mitad, utilizando la ecuación de Debye-Scherrer (11). Los valores obtenidos son característicos de compuestos tipo hidrotalcita con el anión carbonato situado paralelo a las láminas de brucita. Los valores de los parámetros de red son muy similares para un mismo sistema, con independencia de cual haya sido el proceso de envejecimiento aplicado. Los datos de tamaño de partícula indican que el método microondas produce compuestos de menor tamaño de partícula y distribución de tamaños más homogénea. Igualmente, las propiedades de superficie de estos sólidos son muy sensibles al método de síntesis. Los valores de área superficial calculados empleando la ecuación B.E.T, recogidos

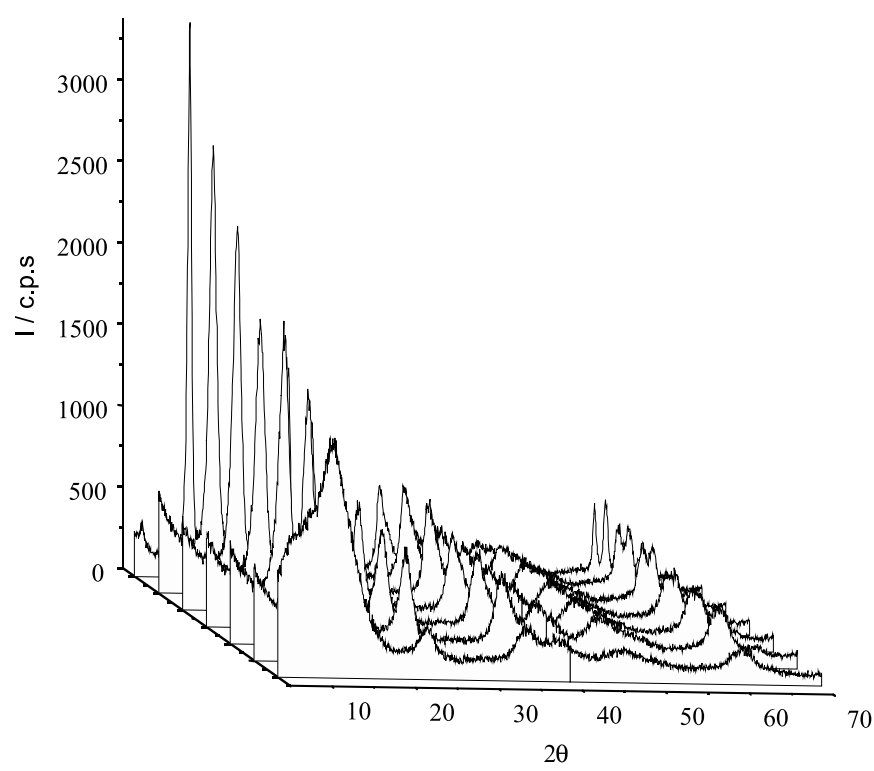

Figura 1. Difractogramas de rayos $\mathrm{X}$ de las muestras del sistema $\mathrm{Mg}, \mathrm{Cr}-\mathrm{CO}_{3}$. De adelante a atrás: $\mathrm{MgCr} 3, \mathrm{MgCrHW} 300, \mathrm{MgCrHW} 180$, MgCrHW60, MgCrHW30, MgCrHW10, MgCr0.

TABLA I. DATOS DE CARACTERIZACIÓN ESTRUCTURAL

\begin{tabular}{|c|c|c|c|c|}
\hline Muestra & $\mathrm{c} / \AA$ & $\mathrm{a} / \AA$ & $\mathrm{D} / \mathrm{nm}$ & $\begin{array}{c}\mathrm{S}_{\text {BET }} \\
/ \mathrm{m}^{2} \mathrm{~g}^{-1}\end{array}$ \\
\hline MgAlHW300 & 22.85 & 3.05 & 17 & 67 \\
\hline MgAl3 & 22.84 & 3.05 & 21 & 21 \\
\hline MgCrHW300 & 22.92 & 3.07 & 8 & 142 \\
\hline MgCr3 & 22.88 & 3.08 & 14 & 86 \\
\hline NiAlHW300 & 23.68 & 3.05 & 8 & 137 \\
\hline NiAl3 & 23.33 & 3.05 & 9 & 110 \\
\hline ZnNiAlHW300 & 22.25 & 3.03 & 21 & 81 \\
\hline ZnNiAl3 & 22.22 & 3.03 & 23 & 50 \\
\hline
\end{tabular}

en la Tabla, son superiores en aquellos compuestos envejecidos en horno microondas, acorde con los tamaños de partícula promedio calculados. La causa de este hecho está en la naturaleza del calentamiento; las microondas producen un calentamiento rápido de toda la suspensión, que favorece la nucleación simultánea de todos los cristales, mientras que en el calentamiento convencional el calor se transmite desde el exterior del recipiente hacia el interior del sólido, por conducción y convección, condicionado por los distintos gradientes térmicos; de este modo, se favorece el crecimiento de los cristales frente a la nucleación (12).

El estudio de estos materiales mediante análisis térmico permite obtener información muy interesantes de la incidencia de la radiación microondas sobre las moléculas de agua interlaminares. En la Figura 2 se recogen los registros de DTA del sistema $\mathrm{Mg}, \mathrm{Cr}-\mathrm{CO}_{3}$ de las muestras envejecidas en horno microondas. El primer efecto endotérmico se puede considerar constituido por dos picos: el registrado a menores temperaturas se atribuye a la eliminación de agua fisisorbida, mientras que el que aparece a temperatura mayor es debido a la eliminación de moléculas de agua interlaminares (13). Seguidamente ocurre la eliminación de los hidroxilos de las láminas en forma de agua y de los carbonatos interlaminares en forma de $\mathrm{CO}_{2}$ (14). Un comportamiento similar se ha registrado para los otros sistemas. Además de estos dos picos intensos, se registra otro efecto endotérmico alrededor de $600^{\circ} \mathrm{C}$, no detectado cuando se registra el análisis en atmósfera de nitrógeno, originado por procesos redox del cromo laminar (15). Un examen más detallado del primer efecto endotérmico revela una evolución de las intensidades de los picos, lo que indica un aumento de la cantidad de agua interlaminar, que se corresponde con una mayor pérdida de peso 
en el estudio termogravimétrico. Este comportamiento se puede atribuir a la interacción de la radiación microondas con el material y, más concretamente, con las especies alojadas entre las láminas (moléculas de agua y aniones carbonato). Asumiendo un estado cuasilíquido en la zona interlaminar y la naturaleza de las microondas, es posible la rotura de los débiles enlaces de hidrógeno presentes y, mediante una reorientación de los dipolos de las moléculas de agua, se conseguiría un mayor orden en el espacio interlaminar, que conduciría a la posible acomodación de moléculas de agua adicionales (16).

En todos los sólidos preparados, el carbonato es el único anión interlaminar, como se deduce del estudio por espectroscopía infrarroja. Los espectros son muy similares para todos los sistemas con independencia de la composición y del método de obtención. Todas las muestras presentan una banda ancha e intensa entre 4000 y $3000 \mathrm{~cm}^{-1}$ debida al modo de tensión de los grupos hidroxilo de las láminas y de las moléculas de agua interlaminar (17), sobre la que se detecta un hombro alrededor de $3000 \mathrm{~cm}^{-1}$, que se adscribe al modo de tensión de grupos hidroxilo de moléculas de agua unidas mediante puentes de hidrógeno a aniones carbonato interlaminares (18). El modo de deformación de moléculas de agua es el responsable de la débil banda a $1640-1620 \mathrm{~cm}^{-1}$. La banda intensa y estrecha a $1375-1365 \mathrm{~cm}^{-1}$ es debida al modo de tensión antisimétrico $v_{3}$ del carbonato interlaminar, desplazado de su posición con respecto al carbonato libre debido al fuerte enlace de hidrógeno con las láminas de hidroxilos y las moléculas de agua (18). Las bandas registradas por debajo de $1000 \mathrm{~cm}^{-1}$ pueden adscribirse al modo $\mathrm{v}_{2}$ del carbonato (alrededor de $850 \mathrm{~cm}^{-1}$ ) y a los modos $\mathrm{M}-\mathrm{OH}$.

\section{CONCLUSIONES}

La radiación microondas, como fuente de energía térmica aplicada a la síntesis de materiales laminares tipo hidrotalcita, conduce a la formación de sólidos de alta cristalinidad y gran homogeneidad en el tamaño de partícula, que presentan mayores valores de superficie específica. Además, el empleo de microondas supone un ahorro de tiempo y de energía comparado con otros métodos convencionales. La naturaleza de la radiación microondas produce un reordenamiento de la zona interlaminar que permite alojar mayor número de moléculas de agua junto al anión.

\section{AGRADECIMIENTOS}

Los autores agradecen la financiación de MCyT (MAT2000-1148C02-01) y de Fundación "Memoria Samuel Solórzano Barruso" de la USAL (ref YORY).

\section{BIBLIOGRAFÍA}

1. V. Rives (editor), Layered Double Hydroxides: Present and Future, Nova Science Publishers, Inc., New York, 2001.

2. C. J. Serna, J. L. Rendón y J. E. Iglesias, “Crystal-chemical study of layered $\left[\mathrm{Al}_{2} \mathrm{Li}(\mathrm{OH})_{6}\right] \mathrm{x} \cdot \mathrm{nH} 2 \mathrm{O}^{\prime \prime}$, Clays Clay Miner., 30, 180-184, 1982.

3. S. Velu, A. Romani, V. Ramasmamy, B. M. Chanda y S. Sivasanker, "Synthesis and physicochemical properties of new hydrotalcite-like anionic clays containing $\mathrm{Zr}(4+)$ in the layers", Stud. Surface Sci. Catal., 118, 941-950, 1998.

4. A. Vaccari, Clays and Catalysis: a promising future, Appl. Clay Sci., 14, 161198, 1999.

5. S. P. Newman y W. Jones, "Synthesis and applications of layered double hydroxides containing organic guests", New J. Chem, 105-115, 1998.

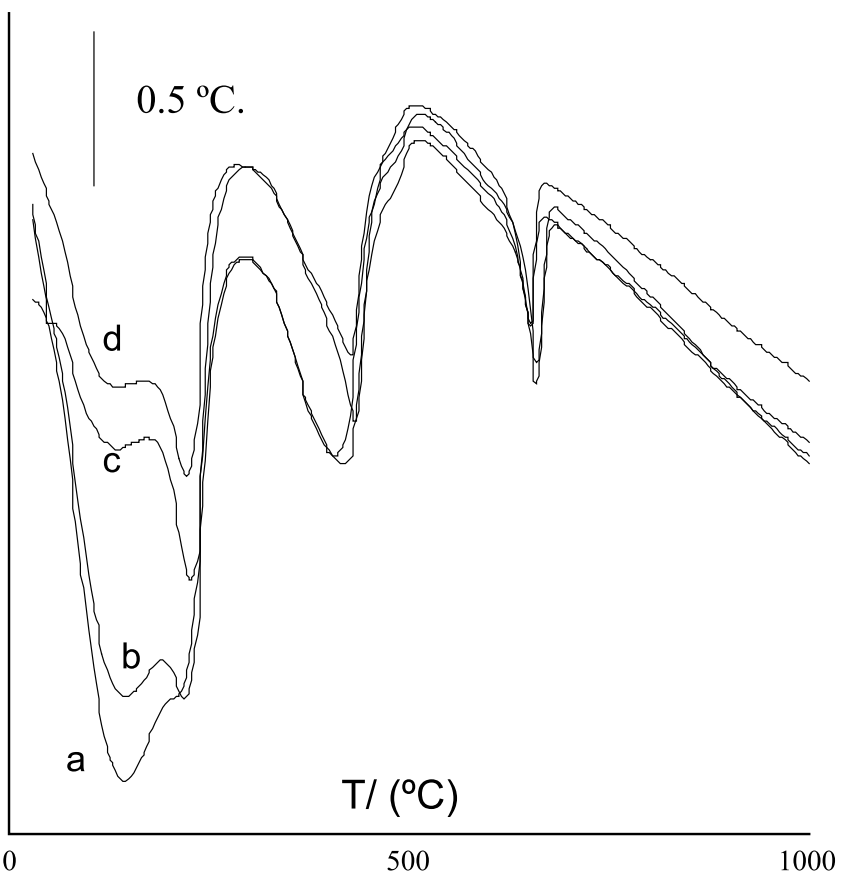

Figura 2. Registros DTA (a) MgCrHW10, (b) MgCrHW60, (c) MgCrHW180, (d) MgCrHW300.

6. V. Rives y M. A. Ulibarri, “Layered Double Hydroxides (LDH) intercalated with metal coordination compounds and oxometalates", Coord. Chem. Rev, 181, 61-120, 1999.

7. F. M. Labajos, V. Rives y M. A. Ulibarri, “Effect of hydrothermal and thermal treatments on the physicochemical properties of $\mathrm{Mg}$-Al hydrotalcite-like materials", J. Mat. Sci, 27, 1546-1552, 1992.

8. K. J. Rao, B. Vaidhyanathan, M Ganguli y P. A. Ramakrishnan, “Synthesis of Inorganic Solids Using Microwaves", Chem. Mat., 11, 882-895, 1999.

9. W. T. Reichle, "Synthesis of Anionic Clay Minerals (Mixed Metal Hydroxides, Hydrotalcite)", Solid State Ionics, 22, 135-141, 1986.

10. A. S. Prakash, P. Vishnu Kamath y M. S. Hedge, "Synthesis and characterization of the layered double hydroxides of $\mathrm{Mg}$ with $\mathrm{Cr}^{\prime \prime}$, Mat. Res. Bull. 35, 2189-2197, 2000.

11. R. Jenkins y J. L. De Vries, Line Broadening, 132-135, Worked Examples in X-Ray Analisis, Second Edition, R. Jenkins y J. L. De Vries, The Macmillan Press Limited, London and Basingstoke, 1978.

12. E. Vileno, Y. Ma, H. Zhou y S.L. Suib, "Facile Synthesis of synthetic todorokite (OMS-1) by co-precipitation reactions in the presence of microwave field", Microporous Mesoporous Mat., 20, 3-15, 1998.

13. V Rives, "Study of Layered Double Hydroxides by Thermal Methods", 115-137, Layered Double Hydroxides: Present and Future, V. Rives (editor), Nova Science Publishers, Inc. New York, 2001.

14. V. Rives, Comment on "Direct Observation of a Metaestable Solid Phase of $\mathrm{Mg} / \mathrm{Al} / \mathrm{CO} 3-L a y e r e d$ Double Hydroxide by Means of High-Temperature in Situ Powder XRD and DTA/TG", Inorg. Chem., 38, 406-407, 1999.

15. F. M. Labajos y V. Rives, "Thermal Evolution of Chromium(III) Ions in Hydrotalcite-like Compounds", Inorg. Chem., 35, 5313-5318, 1996.

16. S. Kannan y R. V. Jasra, "Microwave assisted rapid crystallization of Mg$\mathrm{M}(\mathrm{III})$ hydrotalcite where M(III)=Al, Fe o Cr", J. Mat. Chem, 10, 2311-2314, 2000.

17. M. J. Hernández-Moreno, M. A. Ulibarri, J. L. Rendon y C. J. Serna, IR "Characteristics of Hydrotalcite-like Compounds", Phys. Chem. Minerals, 12, 34-38, 1985.

18. J. T. Kloprogge y R. L. Frost, "Infrared and Raman Spectroscopic Studies of Layered Double Hydroxides (LDHs)", 139-192, Layered Double Hydroxides: Present and Future, V. Rives (editor), Nova Science Publishers, Inc., New York, 2001. 